\title{
TV/Series
}

$5 \mid 2014$

Religions en série

\section{Peut-on être Américain et musulman ? La preuve par l'image dans All-American Muslim}

\section{Nolwenn Mingant}

\section{(2) OpenEdition}

1 Journals

Édition électronique

URL : http://journals.openedition.org/tvseries/449

DOI : $10.4000 /$ tvseries.449

ISSN : 2266-0909

Éditeur

GRIC - Groupe de recherche Identités et Cultures

\section{Référence électronique}

Nolwenn Mingant, "Peut-on être Américain et musulman ? La preuve par l'image dans All-American Muslim », TV/Series [En ligne], 5 | 2014, mis en ligne le 01 mai 2014, consulté le 14 novembre 2019. URL : http://journals.openedition.org/tvseries/449; DOI : 10.4000/tvseries.449

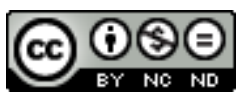

$T V /$ Series est mis à disposition selon les termes de la licence Creative Commons Attribution - Pas d'Utilisation Commerciale - Pas de Modification 4.0 International. 


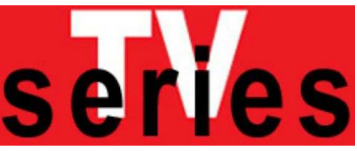

\section{Peut-on être Américain et musulman ? La preuve par l'image dans All-American Muslim}

En 2011, la chaîne commerciale TLC diffuse une série de télé-réalité qui se penche sur la vie de cinq familles musulmanes d'origine libanaise vivant à Dearborn dans le Michigan. Si la chaîne ne présente pas ce programme comme sujet à controverse, All-American Muslim déclenche les passions car elle touche à la représentation d'une minorité dénigrée dans les médias depuis plusieurs décennies et mise au ban de la société depuis les attentats du 11 septembre 2001. Utilisant tous les outils de la télé-réalité, cette série fonctionne comme une démonstration afin d'aller à l'encontre des stéréotypes négatifs de la culture populaire. Premier point: redorer le blason de la religion musulmane. La série démonte systématiquement les images habituelles de l'islam comme une religion violente, oppressive et monolithique. Pour ce faire, la série propose de faire un voyage à l'intérieur de l'islam, plutôt que d'adopter la position extérieure consacrée par plusieurs siècles d'orientalisme. Une fois l'islam présenté comme une religion non-menaçante, la série s'attèle à démontrer l'américanité des participants, insistant sur leur désir d'intégration. Elle les pare des symboles de l'américanité, du barbecue au goût de la consommation, poussant cette revendication identitaire jusqu'à faire de certains participants des « super patriotes ».

If you're following your faith, you're a better citizen.

(Fouad Zanab, 1.1)

$\mathrm{S}$ elon un sondage de 2006, $5 \%$ des Américains pensaient que les musulmans n'étaient pas de loyaux citoyens et $25 \%$ souhaitaient ne pas avoir de voisin musulman ${ }^{1}$. En dépit des discours pacificateurs du président Bush, les actions prises par le gouvernement américain à la suite des attentats du 11 septembre 2001, telle la loi PATRIOT, ont donné un signe fort : les musulmans constituaient une menace pour la société américaine. Sous le coup d'une méfiance généralisée et d'actes de violences, la communauté arabo-musulmane ${ }^{2}$ s'est retrouvée ostracisée. Cette mise au ban de la société a été facilitée par les nombreuses décennies de représentation négative des arabomusulmans dans la culture ${ }^{3}$. Avant le 11 septembre, les clichés principaux étaient le riche scheik, homme barbare et pervers poursuivant de blanches hérö̈nes dans des paysages désertiques et le terroriste, lui aussi pervers, dans la jouissance de la destruction infligée

${ }^{1}$ Louise A. Cainkar, Homeland Insecurity: The Arab American and Muslim American Experiences After 9/11, New York, Russell Sage Foundation, 2009, p. 273.

${ }^{2}$ Dans l'imaginaire américain, il y a une équivalence parfaite entre être Arabe et être musulman. Cette vision monolithique ne prend en compte ni les nombreux Arabes qui ont une autre religion, notamment le catholicisme, ni les musulmans qui ne sont pas arabes (Indonésie, noir-américains).

3 Cainkar, p.66 
au monde occidental ${ }^{4}$. Après le 11 septembre 2001, une nouvelle figure apparait, cette du " voisin arabo-américain terroriste 5 », celui qui sous ses airs anodins prépare le prochain attentat. Cette figure est très présente dans les séries télévisées. Elle est récurrente dans la série 24 heures chrono (Fox Network, 2001-2014); elle est l'objet même de la série Sleeper Cell (Showtime, 2005-), dont le slogan est "Friends. Neighbours. Husbands. Terrorists ${ }^{6}$. " C'est dans ce contexte que la chaîne The Learning Channel (TLC) diffuse en 2011-2012 la série de télé-réalité All-American Muslim ${ }^{7}$. Pendant huit épisodes, la série suit la vie de cinq familles musulmanes d'origine libanaise installées à Dearborn (Michigan), la ville américaine avec la plus forte concentration de musulmans.

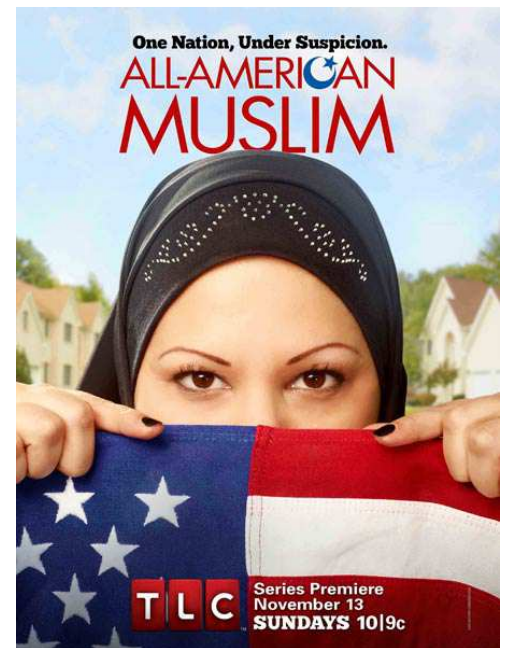

Fig. 1 : affiche publicitaire pour la série avant sa première diffusion en 2011

\footnotetext{
4 Ces stéréotypes sont l'objet des analyses fondatrices de Jack Shaheen. Voir notamment Jack G. Shaheen, The TV Arab, Bowling Green University Popular Press, 1984. Jack Shaheen, Guilt: Hollywood's Verdict on Arabs after 9/11, Northampton: Olive Branch Press, 2008.

5 "The Arab-American Neighbor as Terrorist," Shaheen, Guilty, 47.

${ }^{6}$ L'affiche de Sleeper Cell est visible à cette adresse :

http://www.imdb.com/title/tto465353/, consulté le 2 octobre 2013.

7 All-American Muslim est une série produite par Shed Media US, une entreprise spécialisée dans la télé-réalité. La bande-annonce est disponible sur le site officiel de la série : http://www.tlc.com/tv-shows/all-american-muslim, consulté le 2 octobre 2013.
} 
L'affiche publicitaire (voir figure 1) joue sur ce contexte par une mise en scène très ambiguë8 ${ }^{8}$ En son centre, en gros plan, est placé le visage d'une femme voilée. Des deux mains, elle tient un drapeau américain, ne laissant voir que ses yeux qui regardent le spectateur d'un air à la fois sérieux et provocateur. En arrière-plan, on aperçoit les rangées de maison typiques d'une suburb de la classe moyenne américaine. Cette affiche est d'emblée dérangeante en ce qu'elle ancre une image controversée (celles des femmes voilées) au sein même de la représentation visuelle des Etats-Unis.

Comment interpréter cette affiche? Doit-on s'attendre à un scénario à la Sleeper Cell ? Difficile de croire qu'un programme de téléréalité ait suivi une cellule terroriste. En haut de l'affiche, apparaît le nom de l'émission: All-American Muslim. Là encore, l'ambiguïté règne : les couleurs rouge et bleu renvoient au drapeau américain, mais la lettre « $\mathrm{C}$ » est remplacée par un croissant et une étoile, symbole politique de l'islam. Comment comprendre cette fusion? Sur quelle mode cette lettre s'immisce-t-elle dans le contexte américain? Enfin, le slogan, qui apparaît à l'extrémité supérieure achève de dérouter. Dans une reprise déformée de la devise " One Nation under God » (que l'on pourrait traduire par " une nation sous la protection de Dieu ») du serment d'allégeance au drapeau américain, le poster affiche: «One Nation, Under Suspicion» ("une nation suspecte»). L'affiche joue donc à plein sur le contexte culturel, où l'américanité des musulmans est remise en question. Mais elle n'éclaire pas sur le parti pris de l'émission. Pour le savoir, il faudra regarder TLC, le 13 novembre. Or, dès les premières images de l'émission, le programme culturel apparaît clairement : si ces cinq familles de Dearborn se sont prêtés au jeu (mais peut-on vraiment parler de jeu dans le contexte post-11 septembre), c'est pour mieux parer aux accusations constamment lancées par la société, pour mieux déconstruire les clichés sur l’islam aux États-Unis et pour en proposer une nouvelle vision. Pour cette démonstration, l'émission utilisera toutes les ressources de la télé-réalité, de la scénarisation au montage des images.

\section{Proposer une nouvelle vision de l'islam}

Dès la sortie de l'émission, l'association conservatrice Florida Family lance une campagne de protestation et obtient que certaines entreprises, tel Lowe's, retirent leurs publicités. Selon Florida Family, l'émission est condamnable car elle fait de la « propagande » :

${ }^{8}$ Cette affiche est disponible sur ce lien :

http://www.globaltimes.cn/NEWS/tabid/99/ID/699289/All-American-Muslim-TV-showends-as-viewership-slumps.aspx, consulté le 2 octobre 2013. 
[...] conçue pour aller à l'encontre des inquiétudes actuelles légitimes au sujet des nombreux musulmans qui œuvrent à l'avancement du fondamentalisme islamique et de la Sharia. L'émission ne montre que des musulmans qui semblent être des gens ordinaires tout en excluant les nombreux croyants musulmans dont le programme pose un danger avéré et imminent aux libertés et valeurs traditionnelles que la majorité des Américains chérissent 9 .

Aller à l'encontre de la façon dont les musulmans sont en général présentés dans les médias américains est en effet la ligne conductrice de l'émission. En dehors des types d'images habituels au format de la télé-réalité (scènes de vie filmées et «confessionnal »), All-American Muslim inclut de nombreuses séquences plutôt inspirées des talkshows : assis sur deux grands canapés rouges face à un tapis aux motifs orientaux, les participants discutent et débattent des thèmes principaux de l'épisode (Figure 2). Il s'agit de séquences éminemment didactiques où les participants expliquent leur religion. "Tout ce que vous avez toujours voulu savoir sur les musulmans sans jamais oser le demander » pourrait-on lire en sous-titre. Au fil des huit épisodes, les participants examinent et éclaircirent des points aussi divers que : Qu'est-ce que le Ramadan? Pourquoi l'islam n'autorise pas l'adoption? La Sharia pourrait-elle être appliquée aux États-Unis? Quelle est la différence entre halal et kasher ? Un bon musulman peutil boire de l'alcool et danser ? Pourquoi les musulmans fument-ils le narguilé ? Peut-on se convertir à l'islam ? Pourquoi les musulmans n'aiment-ils pas les chiens ? À travers ces discussions, mais aussi à travers les scènes filmées et montées, ainsi que les moments de " confessionnal», le programme tente donc de répondre aux incompréhensions des Américains. On peut classer ces thèmes divers en trois grands clichés que l'émission se fait fort de déconstruire : l'islam en tant que religion de la violence, de l'oppression et du monolithisme.

9 Florida Family, "The Learning Channel officially cancels All-American Muslim Supporters' emails to advertisers made the difference," mars 2012, http://floridafamily.org/full article.php?article no=108, consulté le 30 septembre 2013. Traduit par l'auteure. 


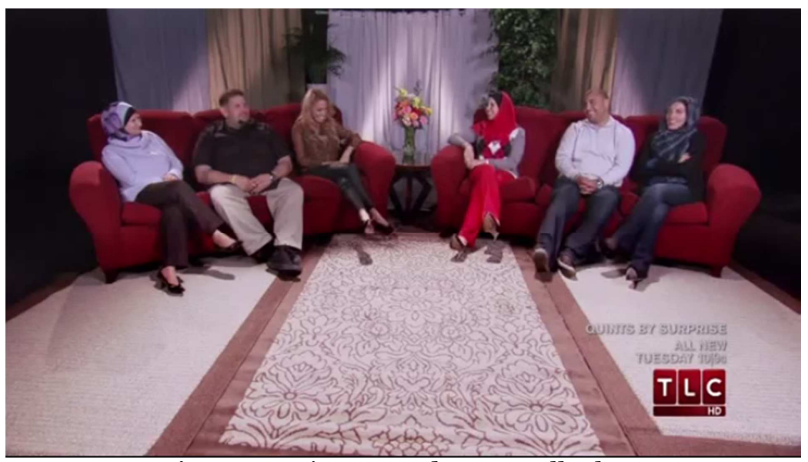

Fig. 2. Les séquences du type talk-show

\section{L’islam : une religion de la violence?}

Pour certains, les attaques du 11 septembre 2001 "viennent confirmer le stéréotype selon lequel les hommes arabes et les musulmans sont intrinsèquement violents ${ }^{10}$ ». Au cinéma, l'image classique du musulman est celle du terroriste hurlant le nom d'Allah avant de faire exploser une bombe. Afin de contrer cette image prédominante accentuée par la circulation d'images montrant des personnes du monde arabe se réjouissant des attentats, la série dédie un épisode entier au 11 septembre, donnant aux participants l'occasion d'exprimer leur point de vue :

Those terrorists attacked us as Americans and hijacked our religion as Muslims. (Suehaila)

I don't feel that the people responsible for $9 / 11$ have the right to be associated with my faith. (Angela)

I don't want to always have to apologize or have to explain myself for these people or defend who I am to people because of these psychos that are out there. (Nawal)

When I first heard it was Muslim extremists that had done something like that, immediately my thoughts like everybody else, every other Muslim's thought was, 'These people are not Muslims. Who are these people? Where do they come from?' I've never heard of the Taliban. I've never heard of Ossama Bin Laden. They've labeled themselves as Muslims but they're not Muslims. A real Muslim would never do anything like that. (...) He [Bin Laden] is just a ... maniac. (...) This is a religion of peace and the fact that this guy came into the picture and wanted to speak on behalf of all Muslims in the world was terrible. It was the worst feeling for a Muslim. He ruined it for us. He ruined it for our kids. (...) He made us scared in our own home. (Nina)

${ }^{10}$ Cainkar, p. 231. 
Ces remarques, exprimées avec beaucoup de vigueur et d'émotion dans l'épisode 7, permettent aux participants de redéfinir leur place dans la logique oppositionnelle du "us versus them » des années Bush" ${ }^{11}$. Le " nous ", c'est la communauté des " vrais musulmans » présentés dans le programme. Ils sont aux antipodes de «eux », les « extrémistes », les terroristes. Le « nous » est alors doublement victime des attentats du 11 septembre : victimes comme tous les Américains de cette attaque contre leur pays, mais aussi victimes de cette attaque sur leur foi. Les participants considèrent de façon unanime que ces extrémistes ne sont pas de vrais musulmans. Pour ceux qui mentionnent que les terroristes ont agi sous couvert de religion, ils estiment que cette religion n'est pas la leur. D'autres rejettent tout à fait l'argument religieux et repoussent les terroristes dans la catégorie des "fous » et "psychopathes ». Ce refus d'être assimilés au pan les plus violents de l'islam est résumé visuellement par le T-Shirt « Not a Terrorist » qu'arbore Shadia dans plusieurs épisodes (Figure 3).

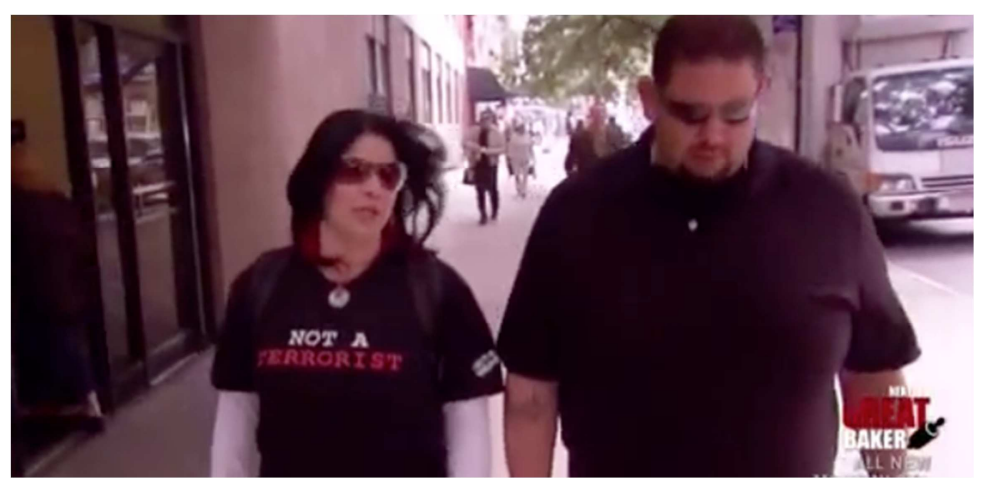

Fig. 3. Le cadrage permet de lire distinctement l'inscription sur le T-shirt de Shadia

Pour dissiper les préjugés, la série ne joue pas seulement sur la parole, sur ces moments où les participants expriment leur opposition aux terroristes. Elle joue également sur la narration et les images, mettant en scène l'islam comme " une religion de paix », vision qui détonne avec la représentation habituelle. Le premier épisode montre ainsi deux cérémonies religieuses ayant lieu dans le foyer des Amen. Chaque cérémonie (une conversion et un mariage) se passe dans une atmosphère familiale, joyeuse et détendue. Les participants font des plaisanteries. Et l'Imam ... rit (Figure 4). La religion est également mise en scène dans des moments émouvants. On voit ainsi Nader se pencher

${ }^{11}$ Georges W. Bush a largement fondé ses discours post-11 septembre sur une dichotomie du "us vs. them" avec d'un côté les Américains et de l'autre les terroristes. 
affectueusement sur le ventre rond de sa femme enceinte et chanter une berceuse à l'enfant à naître. On le verra ensuite prier nerveusement dans le couloir tandis que sa femme accouche, puis murmurer tendrement une bénédiction à l'oreille du nouveau-né (Figure 5). La série insiste enfin sur l'ouverture aux autres religions en montrant les participants, notamment les plus traditionnalistes, participer à des événements œcuméniques, tels Faoud et sa femme se rendant dans une église méthodiste pour une commémoration des attentats du 11 septembre. Au-delà même des déclarations, ces éléments visuels frappent par leur grande efficacité pour proposer une autre vision de l'islam. Associant la religion musulmane à des moments heureux et paisibles, ces images viennent contrecarrer les stéréotypes existants.

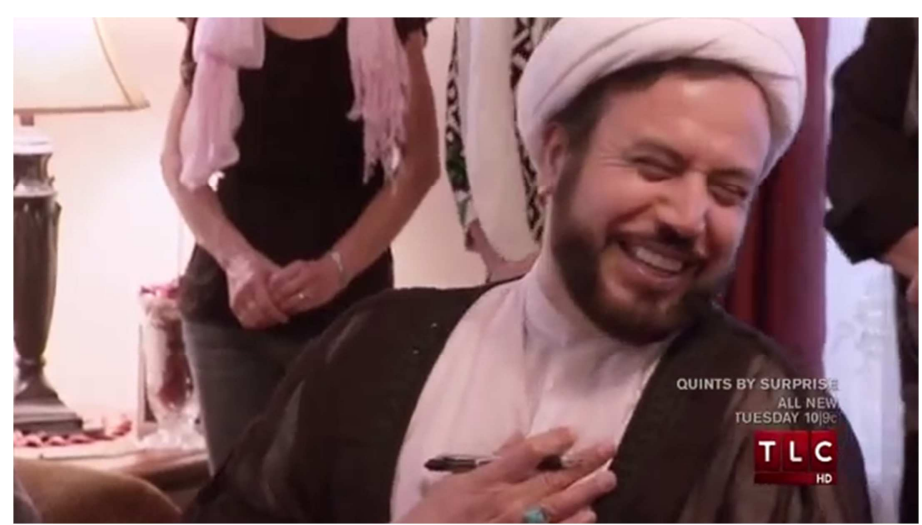

Fig. 4 : L’Imam riant lors de la cérémonie de mariage

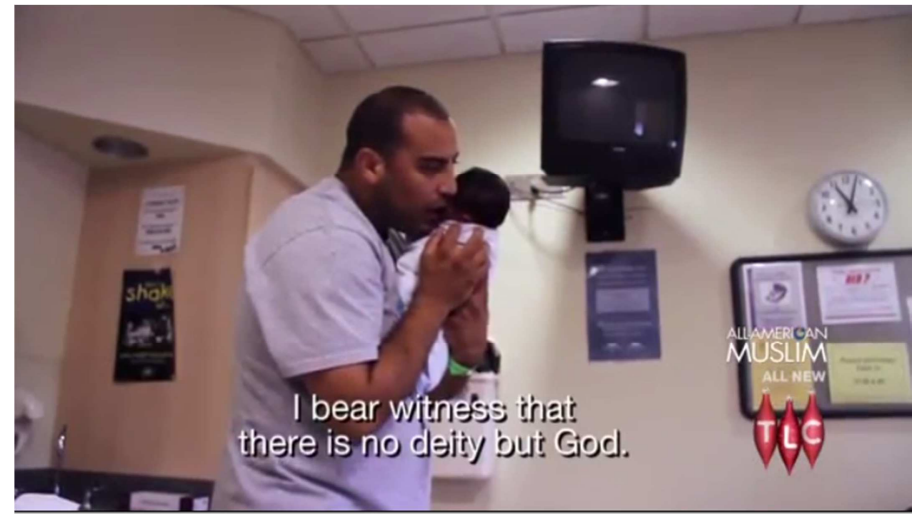

Fig. 5 : Nader murmure une bénédiction à l'oreille de son fils 


\section{L’islam : une religion qui oppresse les femmes?}

Si, dans les médias, les hommes musulmans sont souvent associés à la question de la violence et du terrorisme, les femmes musulmanes sont devenues le symbole de l'oppression religieuse à travers la question du voile. Le hijab est alors vu comme un signe de soumission à une culture patriarcale ${ }^{12}$ dont le caractère autoritaire est perçu comme une violation flagrante des valeurs américaines ${ }^{13}$. La série accorde donc une large place aux questions relatives à la place de la femme dans l'islam : le voile, mais aussi le fait d'être mariée ou non, la pression pour avoir des enfants, le poids de la réputation, l'interdit de serrer la main des hommes, le stigmate du divorce, l'impossibilité d'un mariage mixte ${ }^{14}$. Ici, c'est par le choix des participantes que le programme répond à ces préjugés, car elles ont toutes une identité différente ${ }^{15}$ :

- Zaynad Zaban est mère de quatre enfants et secrétaire à mitemps. Traditionnaliste, elle porte le voile, ainsi que ses filles.

- Samira Amen ne porte pas le voile au début de la série. Cependant, n'arrivant pas à avoir d'enfant, elle décide de porter le voile afin de faire un geste fort de dévotion religieuse. Elle est secrétaire médicale.

- Shadia Amen est une jeune rebelle. Non voilée, elle arbore des tatouages. Elle est fiancée à un jeune homme d'origine irlandaise. Elle a un fils, issu de son premier mariage.

- Angela Jaafar est mère de quatre enfants. Elle ne porte pas de voile et a une position progressiste. C'est une femme d'affaire très impliquée dans son travail.

- Nawal Aoude est une jeune mariée qui attend un enfant. Elle est voilée et plutôt traditionnaliste. Elle travaille comme inhalothérapeute.

- Nina Bazzy est mère d'un enfant. Elle n'est pas voilée. Elle a un look qu'on peut qualifier de bimbo : cheveux longs teints en blond, jambes longues et forte poitrine, moulée dans des tenues sexy. Elle est organisatrice de mariages et souhaite ouvrir une boîte de nuit.

À travers ce choix de participantes, la série assène plusieurs faits. Le plus important est que le voile n'est pas une obligation. Il est d'ailleurs

\footnotetext{
${ }_{12}$ Evelyn Alsultany, Arabs and Muslims in the Media: Race and Representation after 9/11, New York, New York University Press, 2012, p. 71.

${ }_{13}$ Cainkar, p. 233

${ }^{14}$ Le public de TLC est majoritairement féminin, ce qui explique également l'importance de ces thèmes.

15 On peut voir les photos de ces différentes femmes sur le site de l'émission: http://www.tlc.com/tv-shows/all-american-muslim, consulté le 2 octobre 2013.
} 
souvent présenté comme un choix de la part des participantes. Par ailleurs, ces femmes ne sont pas exclues de toute vie sociale. Ce sont en effet toutes des femmes qui travaillent hors de chez elles. Le voile ne semble d'ailleurs pas être un obstacle à l'entrée sur le marché du travail. Ainsi, lorsque Samira arrive voilée au travail, elle est accueillie de façon positive par ses collègues dont la seule réaction (du moins celle enregistrée sur pellicule) est que le voile lui sied.

Le personnage le plus représentatif de ce que la série semble vouloir promouvoir est Suehaila Amen. Très ancrée dans la tradition, elle porte le voile. Célibataire bien qu'ayant la trentaine, elle vit chez ses parents. Cependant, c'est également une jeune femme très active. Son voile ne l'empêche pas de travailler comme greffier. Les épisodes la montrent d'ailleurs fréquemment dans son environnement de travail. Elle est également très engagée pour la défense des droits des musulmans américains, n'hésitant pas à prendre l'avion seule pour faire une conférence à Washington. On la voit même danser dans une soirée organisée par Nina. Elle semble représenter la fusion idéale entre un dynamisme et un engagement typiquement américains, et un respect des valeurs musulmanes.

Au-delà des sessions de talk-show où les femmes disent porter le voile par choix, pour préserver leur modestie, la diversité de ces femmes, mais surtout leur caractère actif, va à l'encontre du cliché du "tas de linge noir", celui de la femme soumise en burka qu'évoque Shaheen pour le cinéma ${ }^{16}$. Le bilan représentationnel est cependant plus mitigé que sur la question de la violence terroriste. En effet, plusieurs participantes très différentes expriment parfois un malaise : le poids de la tradition est un obstacle tant pour la blonde et sexy Nina qui souhaite ouvrir une boîte de nuit, que pour la célibataire voilée Suehaila qui voudrait prendre un appartement seule dans une autre ville. Ces contraintes apparaissent cependant liées à un conservatisme social qui n'est pas sans rappeler celui existant dans d'autres communautés très influencées par la religion, qu'il s'agisse du catholicisme des immigrés latino-américains ou de l'évangélisme du Sud des Etats-Unis.

\section{L'islam : une religion monolithique?}

Cette large palette de participantes féminines participe également à mettre à mal un autre cliché, celui d'un islam monolithique, peuplé uniquement de fondamentalistes. Ici, l'importance du choix de séances de type talk-show apparaît comme capitale: elles offrent une opportunité didactique et, surtout, elles montrent des musulmans qui interprètent leur religion sans être toujours d'accord. Une des lignes

${ }^{16}$ Shaheen parle de 'Bundles of Black.' Jack G. Shaheen, Reel Bad Arabs: How Hollywood Vilifies a People, Northampton: Olive Branch Press, 2009 [2001]), p. 28. 
narratives mises en scène par les réalisateurs met en avant cette diversité. Au moment du Ramadan, Fouad, l'entraîneur de l'équipe de football du lycée, décide que les entraînements auront lieu la nuit afin que les jeunes joueurs puissent manger et boire au moment de l'effort physique. Cette histoire se déroule entre l'épisode 2 et 6 et prend une place importante tant dans les scènes narratives que dans les scènes de confessionnal. La série entretient le suspense : les parents des joueurs non-musulmans vont-ils s'inquiéter? Les jeunes vont-ils tenir ? Et, surtout, vont-ils réussir à gagner un match important pendant le Ramadan ? C'est l'occasion de montrer la diversité des courants de la communauté. Fouad, présenté comme traditionnaliste, est soutenu par certains participants comme Nader. À l'inverse, Mike, le shérif, déclare trouver ces entraînements de nuit... franchement absurdes. Tout au long de la série, les familles de Mike et de Fouad sont d'ailleurs présentées comme opposées. L'épisode 5 qui est consacré à la rentrée des classes montre des préoccupations très différentes : la femme de Fouad s'inquiète des hijab de ses filles, tandis que celle de Mike s'inquiète de pouvoir concilier ses obligations familiales et professionnelles. $\mathrm{Au}$ traditionnalisme d'une famille répond le progressisme de l'autre. Par ce choix de participants vivant leur foi de façon différente, par la mise en scène d'épisodes montrant ces différences, la série individualise les musulmans. Le spectateur, qui s'est attaché à tel personnage, qui s'est pris d'antipathie pour tel autre, ne peut plus dès lors continuer d'adhérer à la vision essentialisante traditionnellement véhiculée dans les médias.

\section{L’islam : la religion de l'Autre}

Si un processus d'essentialisation a pu avoir lieu, c'est que la religion musulmane a été traditionnellement considérée de l'extérieur, dans le cadre de l'orientalisme, cette vision de l'Orient fantasmée et construite par l'Occident, depuis les premiers récits de voyage des aventuriers littéraires européens ${ }^{17}$. Ici, All-American Muslim renverse le dispositif habituel du regard, et ce dès les premières scènes. Environ sept minutes après le début de l'épisode pilote, l'on voit des musulmans en train de prier. Les premières images sont proches des représentations habituelles: la mosquée est vue en plan large, accompagnée d'une bande-son constituée de psalmodie. Un plan large nous montre un groupe indifférencié d'hommes agenouillés (Figure 6). La caméra positionnée en hauteur et placée derrière les hommes propose donc une vue en plongée habituelle des points de vue extérieurs, supérieurs voire ethnographiques, présents dans le cinéma hollywoodien. Mais ce schéma représentationnel est immédiatement mis à mal par les plans suivants : un plan large de la maison des Amen,

${ }_{17}$ Voir Edward W. Said, Orientalism, Londres, Penguin Books, 2003 [1978]. 
un gros plan sur Bilal priant dans son salon debout et non plus prosterné (Figure 7), puis sur son père priant dans le jardin. La bande son n'est plus une psalmodie lointaine et indifférenciée, mais la voix de Bilal qui explique que, pour lui, prier est un signe de respect pour Dieu. Ces images déplacent radicalement le champ de vision : de l'extérieur vers l'intérieur, du lointain vers le proche. C'est en effet une plongée dans l'intimité de ces familles que l'émission propose. Il ne s'agit pas d'être un voyageur qui reste sur le pas de la porte ; il faut entrer dans les maisons et s'ouvrir à la culture des habitants.

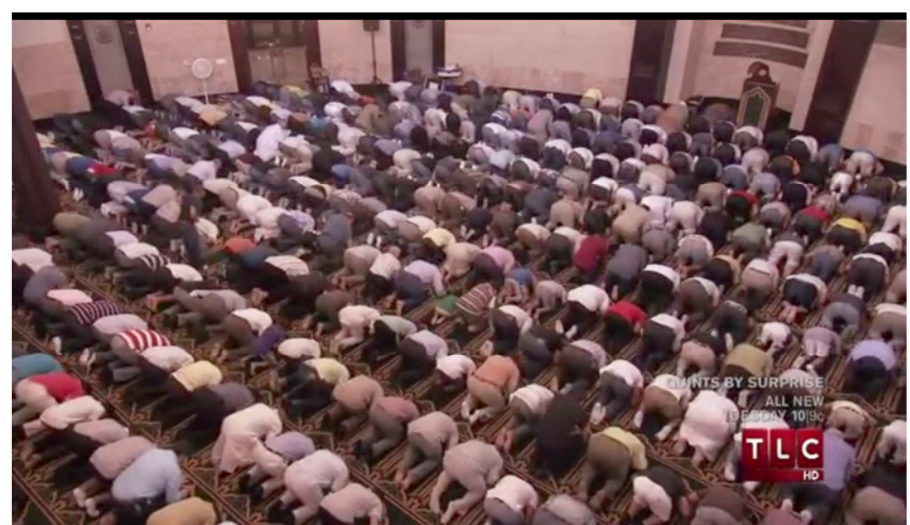

Fig. 6. Un groupe indifférencié prie à la mosquée.

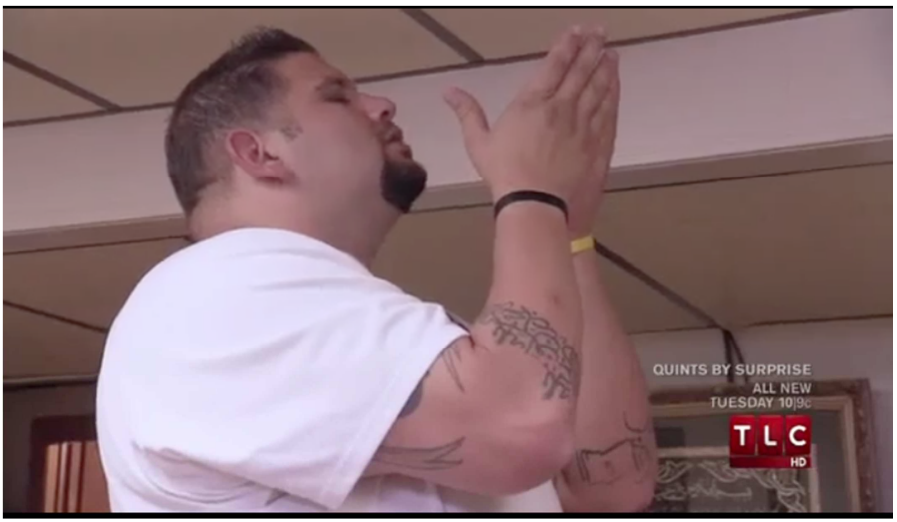

Fig. 7. Bilal prie dans son salon.

Pour le spectateur non-musulman, la série propose même une figure qui pourra servir de guide, auquel il pourra s'identifier. Coup de chance ou coup de génie, l'un des participants est en effet un jeune 
Américain d'origine irlandaise et catholique. Afin de pouvoir épouser sa bien-aimée, Shadia, Jeff McDermott se convertit à l'islam au premier épisode. Ce participant fera pendant toute la série office de passeur. Son importance symbolique est très forte puisqu'il est celui qui va vers l'Autre, qui fait de la religion de l'Autre la sienne. Cette conversion est montrée sous un jour très positif. Jeff vient vers l'islam non par rejet de sa communauté d'origine, mais par amour pour Shadia. Si sa mère est attristée par ce choix, elle est néanmoins à la cérémonie. Toute la famille est présente à la fête de mariage. L'inclusion spatiale traduit l'inclusion émotionnelle. Les cérémonies de conversion et de mariage ont lieu dans l'intimité des Amen, dans l'espace clôt de leur salon. Le choix de cadrages serrés (Figures 8 et 9) accentue cette impression d'une communauté soudée, communauté où Jeff trouve toute sa place. «Now, I have two families. » explique-t-il, serein.

Allant à l'encontre des représentations traditionnellement présentes dans les médias, la série de télé-réalité All-American Muslim utilise donc pleinement toutes les techniques à sa disposition (séquences didactiques, images marquantes, choix des participants, mise en récit) afin de donner une vision non-menaçante de la religion musulmane. Elle la montre de l'intérieur comme une religion tout à fait ordinaire, paisible et diverse. Loin d'être des extrémistes, les participants paraissent alors bien banals, vivant une vie quotidienne quelconque. Ces musulmans pourraient-ils alors être aussi des Américains moyens?

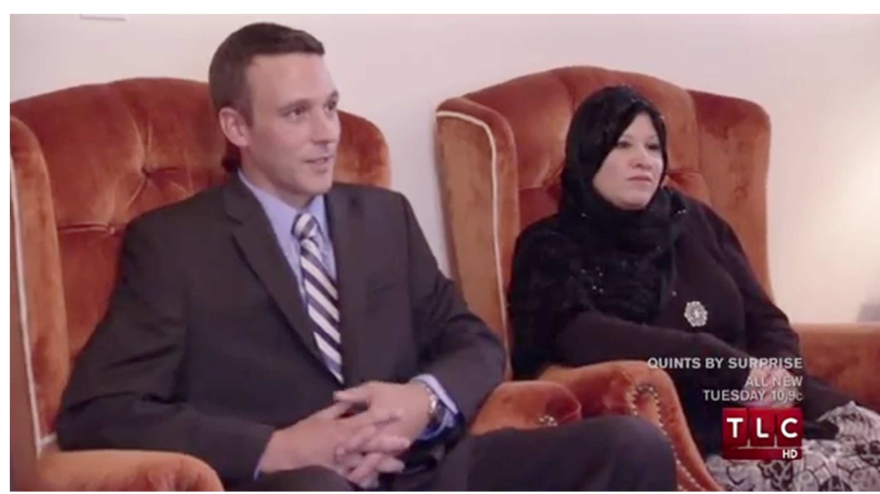

Fig. 8. Jeff et Shadia au moment de la cérémonie du mariage 


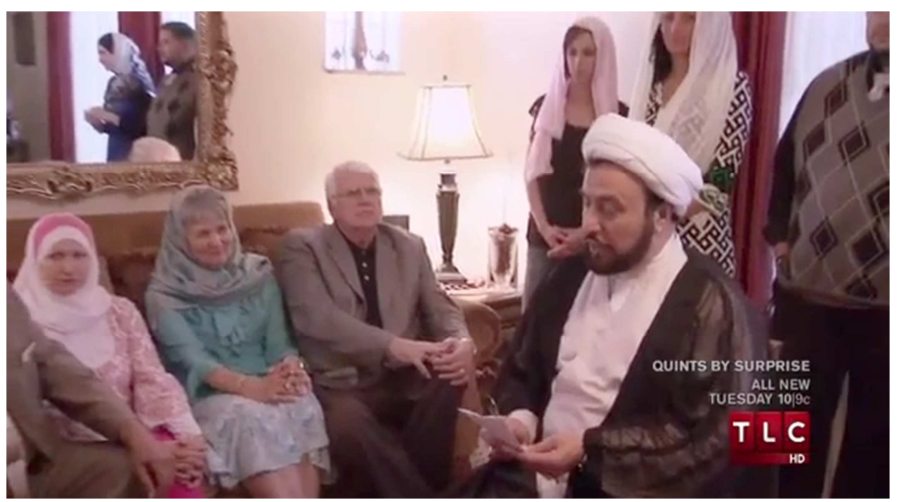

Fig. 9. L’Imam célèbre le mariage en présence de la famille.

\section{Américains, musulmans... et fiers de l'être}

\section{Loin de l'intégrisme... l’intégration}

$\mathrm{Au}$ moment de la sortie de la série, le journaliste et animateur de télévision Anderson Cooper reçoit deux couples de participants du programme dans son talkshow Anderson Live. Dans le public, une femme nommée Mélanie, qui milite pour l'annulation du programme, réagit fortement. Lorsque Cooper lui demande si, selon elle, il est possible d'être « un bon musulman et un bon Américain », elle répond par la négative ${ }^{18}$. C'est à cette apparente incompatibilité que le programme s'attaque, en donnant une vision non-menaçante de l'islam, mais également en mettant en valeur les caractéristiques typiquement américaines des participants. Ce qui frappe par exemple dès le premier épisode est la quasi-absence de la langue arabe. Les participants au centre des histoires parlent tous anglais avec un fort accent... américain. Loin des intégristes hurlant des imprécations en arabe, le programme donne à voir des citoyens américains. La langue arabe est entendue au moment des prières, mais, prononcée sur un ton doux, elle participe à cette description d'une religion paisible que nous avons mentionnée plus haut. Cependant, même dans les contextes religieux, l'arabe laisse largement la place à l'anglais. Entamant la cérémonie de mariage de Jeff et Shadia, l'Imam s'exclame ainsi d'un ton jovial: " Tout le monde parle anglais ici, pas besoin de parler arabe » (épisode 1). La relation des participants à la langue arabe est en fait assimilée à celle des immigrés, particulièrement hispaniques, à leur

\footnotetext{
${ }^{18}$ Davidson, Amy, "The attack on 'All-American Muslim », New Yorker.com, 14 décembre 2011, http://www.newyorker.com/online/blogs/comment/2011/12/the-attack-on-allamerican-muslim.html\#ixzz2CIlMVxGZ, consulté le 30 septembre 2013.
} 
langue d'origine. En effet, si les participants parlent anglais, leurs parents parlent arabe. Se dessine donc une différence entre première et deuxième générations, accentuant l'idée d'une assimilation progressive. Les jeunes générations émaillent leurs phrases de termes arabes («Inchallah», «halal»), dans un équivalent arabe du Spanglish, cette contraction de l'anglais et de l'espagnol utilisé par les immigrés hispaniques de deuxième génération. Analysant deux campagnes de relations publiques de l'année 2003, Evelyn Alsultany note que pour montrer les compatibilités entre américanité et islam, les créateurs ont développé la notion de " patriotisme de la diversité » (diversity patriotism) : " une version du patriotisme américain qui glorifie la notion de citoyenneté diverse et insiste sur l'unité multiculturelle de l'Amérique ${ }^{19}$. " Les connotations liées aux pratiques linguistiques dans All-American Muslim renvoient bien à cette intégration des musulmans dans une société américaine diverse, au même titre que d'autres communautés d'origine immigrée.

La question de l'intégration est également mise en récit par l'histoire entre Jeff, le jeune homme d'origine irlandaise, et Shadia, la jeune femme d'origine libanaise. Ici, c'est l'amour qui fait œuvre d'intégration. Leur mariage, qui a lieu dans le premier épisode, vient montrer visuellement que deux communautés peuvent se retrouver, tandis que danse irlandaise traditionnelle et danse du ventre alternent. Cette fusion est rappelée constamment, par d'infimes détails, comme lorsque Shadia cuisine un plat irlandais à base de bœuf bouilli et de chou ... mais en version halal (épisode 8). Si l'union de deux cultures est présentée comme possible et désirable, l'histoire du chien de Jeff (épisodes 5 et 6) vient montrer au spectateur que l'intégration ne va pas de soi et qu'elle demande une forte volonté. Au moment d'aménager dans leur nouvelle maison, Shadia demande à Jeff de se séparer de son chien, notamment parce qu'il met son père mal à l'aise, ces animaux étant considérés comme impurs dans la religion musulmane. Jeff accepte à contre-cœur. Devant la tristesse du jeune homme, la mère de Shadia intervient. Insistant sur les nombreux efforts qu'il a consentis pour leur couple (conversion à l'islam, tentative de respect du Ramadan), elle lui conseille de reprendre le chien. Shadia cède et, le chien revenu, le couple retrouve l'harmonie. Cette ligne narrative, qui occupe deux épisodes, est loin d'être anecdotique. Dans

19 Alsultnay, p. 134. Alsultany présente deux campages, l'une publique intitulée «I am an American », l'autre privée de l'association CAIR intitulée «I am an American Muslim. » Nous reviendrons sur cette dernière qui présente des points de comparaison frappants avec le programme de télé-réalité étudié ici. Cette campagne a été très peu diffusée et seule une affiche est visible sur internet, au bas de cette page : http://www.asjournal.org/archive/52/156.html, consulté le 2 octobre 2013. Nous nous appuierons donc sur l'étude menée par Alsultany. Les affiches qu'elle analyse sont également visibles dans son article "Selling American Diversity and Muslim American Identiy through Nonprofit Advertising Post-9/11 », American Quarterly, vol.59, $\mathrm{n}^{\circ} 3$, September 2007, pp.93-622. 
leur chapitre intitulé « Faut-il sauver tous les chiens ? ", Vincent Amiel et Pascal Couté analysent le rôle de cet animal dans les fictions américaines. D'un point de vue symbolique, il révèle l'humanité du héros et renforce le lien avec le spectateur, en inscrivant la fiction dans son univers quotidien ${ }^{20}$. En chassant le chien, en partie pour des raisons religieuses, Shalia lui refuse cette fonction. L'insistance du montage sur la tristesse de Jeff place d'ailleurs le spectateur fermement du côté du jeune homme. Le retour de l'animal est présenté de façon très positive. En acceptant le chien, Shadia fait sienne l'image traditionnelle et stéréotypée du foyer américain. L'intégration dans le rêve américain demande des efforts et des compromis que les participants (et avec eux tous les musulmans, semble indiquer la série) sont prêts à faire.

Il est d'ailleurs intéressant que ce mariage mixte se fasse avec un jeune homme d'origine irlandaise et catholique. Lorsque Mélanie, la militante de l'émission d'Anderson Cooper, affirme qu'on ne peut être Américain et musulman, elle explique :

Je ne dis pas qu'ils ne peuvent pas être Américains. Mais alors, ce que je dis c'est que ne sont pas de vrais musulmans. Alors, je veux dire, je voudrais leur poser cette question : Vivez-vous selon le Coran, vivezvous selon les principes du prophète Mahomet faites-vous ce que l'on vous commande de faire ${ }^{21}$ ?

Ce soupçon selon lequel les musulmans américains répondraient à des ordres supérieurs venus de l'étranger, obéissant à la Sharia plutôt qu'à la Constitution, rappelle fortement les reproches faits aux catholiques au milieu du XIX Xiècle $^{22}$ et si cette histoire lointaine n'est pas forcément présente à l'esprit des téléspectateurs, pour autant, Jeff n'est pas WASP, rappelant que l'intégration des immigrés s'est faite par vagues. $\mathrm{Si}$ les catholiques ne sont plus maintenant objets de discrimination aux États-Unis, ce sera un jour le tour des musulmans.

Ce désir d'intégration, cette volonté d'être un vrai Américain sont d'ailleurs symbolisés par les métiers exercés par les participants. On peut à nouveau ici établir un parallèle avec la campagne de relation publique «I am an American Muslim » lancée par l'organisme Council on American-Islamic Relations (CAIR) en 2003. Alsultany note la façon dont les discours de cette campagne « incluent les musulmans au sein de la citoyenneté culturelle américaine » :

${ }^{20}$ Vincent, Amiel, Pascal, Coute, Formes et obsessions du cinéma américain contemporain (1980-2002), Paris, Klincksieck, 2003, p.144-5.

${ }^{21}$ Citée dans Davidson.

${ }_{22}$ Roger, Daniels, Coming to America: a History of Immigration and Ethnicity in American Life, Ney York, Harper Perennial, 2002 [1990], p.267. 
Le service à la nation s'exprime pour les hommes dans le service militaire comme la mise en œuvre parfaite du patriotisme, et pour les femmes par le fait d'être GirlScout ou de faire du bénévolat ou de l'humanitaire 23 .

La grande majorité des participants de All-American Muslim ont un travail correspondant à ce descriptif. Plusieurs participants sont des employés de l'Etat : Mike est shérif, Nader est agent fédéral, Suehaila est greffière. D’autres exercent des métiers dans le domaine de la santé (inhalopthérapeute, secrétaire médicale) ou de l'éducation (assistante dans une école, responsable d'une organisation sportive pour la jeunesse). Ce sont donc soit des métiers représentant l'autorité, soit des métiers tournés vers les autres. Dans les deux cas, la notion de service aux autres et à la nation est centrale. Par leurs choix de carrière, ces musulmans sont des citoyens qui contribuent activement au bienêtre de la société américaine.

Enfin, l'intégration est culturelle. Non seulement les participants sont pétris de culture américaine, mais ils en deviennent des figures symboliques. Shadia représente la rebelle, avec ses tatouages et son amour de la musique country, dans une sorte de figure sage de la contre-culture. Nawal est la fashionista. Elle le reconnaît elle-même et une brève image vient accentuer cette idée. Son placard à chaussures, entraperçu brièvement dans l'épisode 8 (Figure 10) rappelle des images similaires dans des séries de téléréalité telle Keeping up with the Kardashians (E !, 2007-) mais aussi des séries de fiction célébrant la société de consommation, telles Sex and the City (HBO, 1998-2004) ou Desperate Housewives (ABC, 2004-2012). Nina, la blonde pulpeuse, devient le symbole du self-made (wo)man. Sa volonté d'ouvrir une boîte de nuit dans un quartier musulman montre sa détermination et son esprit d'entreprise. Elle est constamment décrite comme une personne forte, qui a du cran. Les nombreux obstacles sur sa route ne font pas fléchir sa volonté de réaliser son rêve.

23 Alstultany, p. 142. 


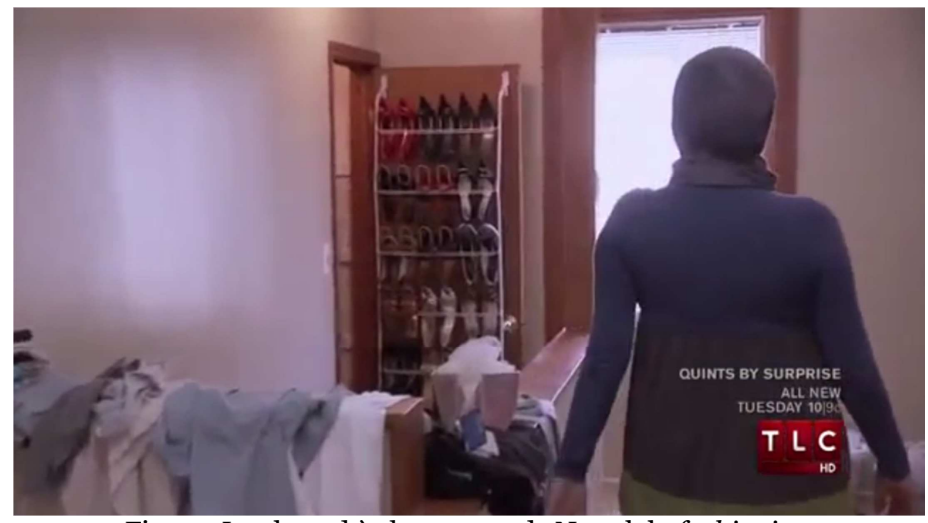

Fig. 10. Le placard à chaussures de Nawal, la fashionista

Pas intégristes, intégrés, et désireux de l'être, voilà le message de la série, message qui apparaît clairement sur le site internet. Sous l'intitulé «Faites connaissance avec les familles » (Meet the Families), sont placées cinq photographies de groupe, une par famille ${ }^{24}$. Les familles posent devant leur maison, les pieds solidement campés sur leur pelouse impeccable. Les participants regardent l'objectif, le sourire aux lèvres. Les parents posent de façon protectrice leur main sur l'épaule des enfants. Les familles apparaissent comme soudées, photographiées par groupe serré, centré au milieu d'un plan large. Ces photos renvoient à des clichés établis : celui de la famille de classe moyenne devant son pavillon de banlieue, incarnation d'un certain rêve américain (Figure 11). On retrouve la même iconographie dans la campagne de publicité de CAIR. Cependant, la télé-réalité permet d'aller au-delà de l'image extérieure. En faisant rentrer le spectateur dans l'intimité des familles, All-American Muslim démontre que cette américanité n'est pas qu'une façade, qu'elle est bien réelle. Derrière cette image d'Epinal ne se cachent pas des étrangers ou des cellules dormantes, mais bien des Américains moyens; pas des "voisins arabo-américains terroristes » mais de simples next-door neighbours.

${ }^{24}$ Site officiel de la série : http://www.tlc.com/tv-shows/all-american-muslim/about-theshow/all-american-muslim-families.htm, consulté le 2octobre 2013. 


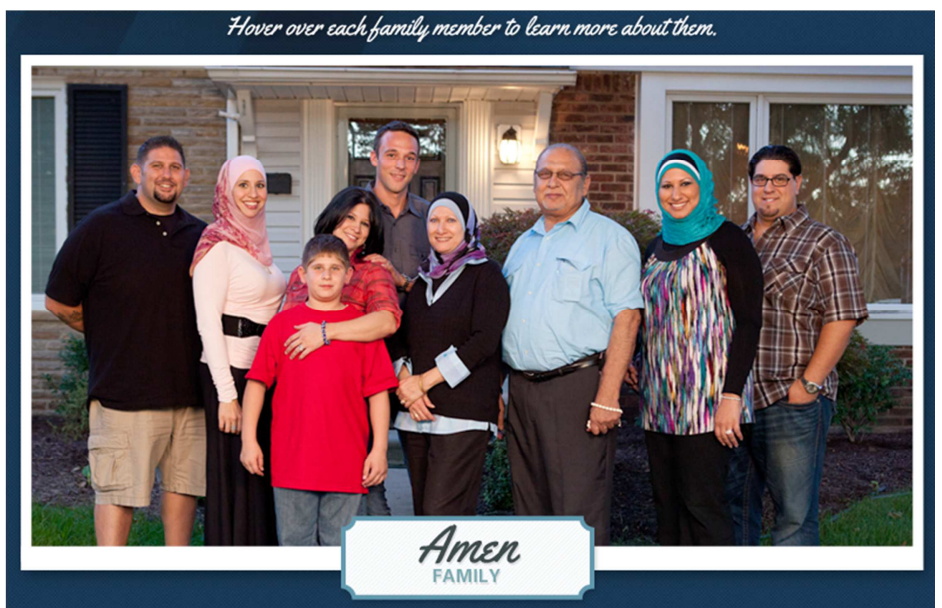

Figure 11. Photographie de la famille Amen (Site officiel de la série)

\section{Fouad Zanab, le super-patriote}

Un participant (ou devrait-on dire «personnage » vu le degré de scénarisation évident de la série) rassemble toutes ces qualités, incarnant la figure idéalisée du musulman américain. Il s'agit de Fouad Zanab, l'entraîneur de l'équipe de football du lycée de Fodson. La majorité des épisodes font la part belle aux scènes d'entraînement sur le terrain. Ce sport représente la quintessence de l'Amérique, jeune, saine et battante. On peut alors considérer le terrain de football comme une métaphore du territoire américain ${ }^{25}$, sur lequel des équipes multiculturelles se mesurent. Les images du terrain incluent d'ailleurs très souvent dans leur cadre le drapeau américain (Figure 12). Dans cette lecture allégorique, Fouad devient la figure même de l'Amérique.

${ }^{25}$ On se rappellera que dans le film américano-indien My Name is Khan (K. Johar, 2010), c'est sur un terrain de football que le jeune enfant de l'héroïne est battu à mort, événement tragique traduisant la déliquescence morale de l'Amérique post-11 septembre, la perte de cette tolérance multiculturelle censée faire sa spécificité et sa force. 


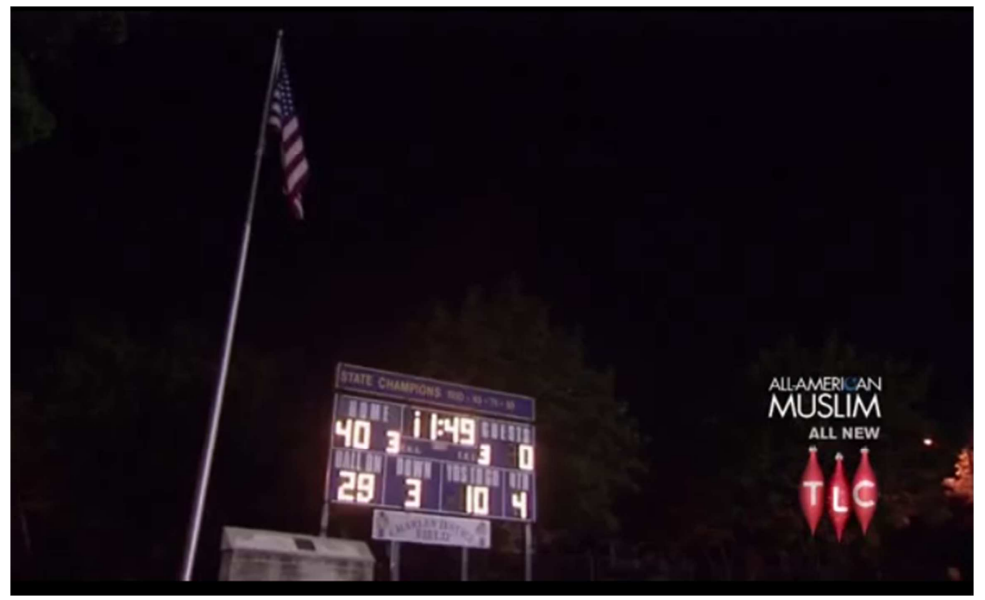

Fig. 12. Le drapeau américain flotte sur le terrain de football.

De fait, Fouad est toujours cadré de façon valorisante. Dans la figure 13 ci-dessous, il se détache ainsi par rapport au groupe indistinct des joueurs, groupe situé plus bas que lui. Il est celui qui donne les ordres, qui maîtrise la situation.

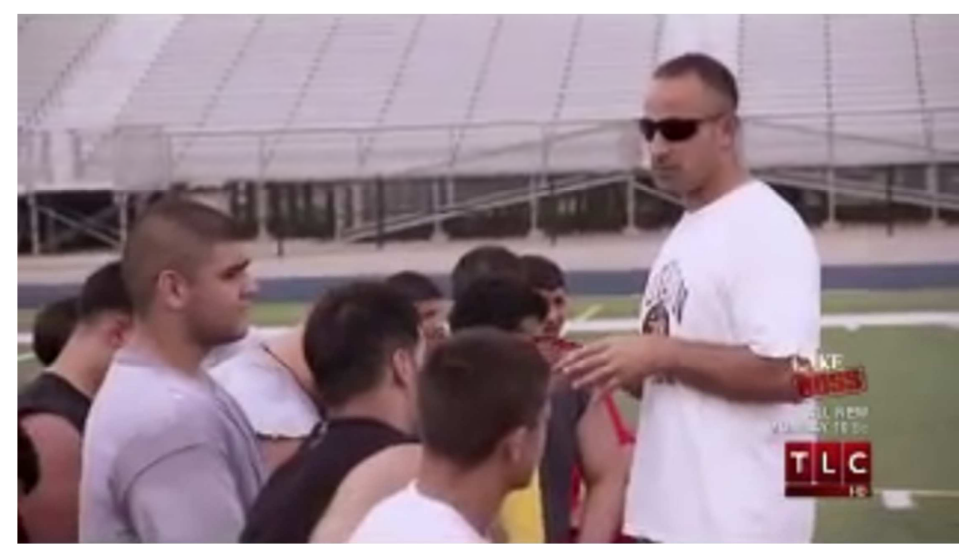

Fig. 13. Fouad donne ses conseils à son équipe

De la même manière, la rencontre avec les parents d'un de ses joueurs le place en position de supériorité (Figures 14 et 15). La caméra nous montre Fouad confortablement installé, droit dans son fauteuil, dans une très légère contre-plongée valorisante. À l'inverse, les parents semblent assis sur le bord d'un banc, un peu courbés. Ils sont en 
position de demandeur et une caméra légèrement plongeante contribue à les mettre en position d'infériorité.

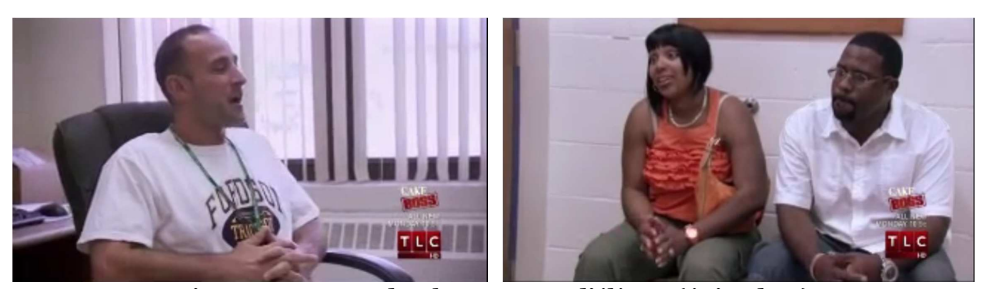

Figs. 14-15. Fouad et les parents d'élèves (épisode 2)

Si de tels cadrages font de Fouad une figure d'autorité, il est aussi présenté comme un personnage dévoué au service des jeunes. Ainsi, au cours du troisième épisode, le spectateur a plusieurs fois l'occasion d'entendre que Fouad n'a jamais manqué un seul entraînement en 17 ans. Ce service rendu à la communauté est d'ailleurs reconnu officiellement lorsque Fouad est invité à la Maison Blanche pour le dîner d'Iftar du Président ${ }^{26}$. De façon très significative, les réalisateurs ont choisi d'intituler cet épisode : "A Muslim Goes to Washington ». Cette référence transparente au film de Frank Capra Mr. Smith Goes to Washington (Mr. Smith au Sénat, 1939), opère une mise en parallèle. Ce sont Dearborn et Fouad qui incarnent désormais les valeurs pastorales de la petite ville américaine (small-town America), tels la famille, le respect de la tradition et le bon sens. Touche suprême pour affirmer l'américanité du coach: une scène le présente en train de cuisiner pour sa famille... au barbecue. Ce participant qui pousse l'américanité à son degré le plus élevé devient un "super-patriote » (« über-patriot $27 »$ ).

Mais si Fouad est 100\% Américain, il est aussi 100\% musulman. En effet, c'est l'un des personnages les plus zélés de la série, celui qui a l'idée des entraînements de nuit pendant le Ramadan, celui dont la femme et les filles sont voilées, celui qui exprime les idées les plus conservatrices lors des séances de talk-show. Là encore, les choix opérés par les concepteurs de la série offrent une forte résonnance avec les publicités de CAIR. Ces publicités tendent à se placer sur le terrain des valeurs morales. Elles insistent notamment sur la notion de valeurs familiales (family values), d’hétérosexualité et de sécurité nationale :

Ces publicités visent à toucher les autres Américains qui ont des valeurs religieuses, vraisemblablement la majorité des Américains, et, ce

${ }^{26}$ L'épisode 3 repose sur le dilemme suivant : Fouad manquera-t-il un entraînement (ce qui n'est jamais arrivé en 17 ans, rappelons-le) pour aller au dîner à la Maison Blanche? Comment choisir entre ces deux lieux symboles de l'américanité ?

${ }^{27}$ Alsultany, p. 148. 
faisant, elles cherchent à placer l'islam sur le même niveau et dans la même structure de valeur que les autres fois, en particulier le christianisme et le judaïsme ${ }^{28}$.

Tout comme les publicités de CAIR, All-American Muslim, en particulier en mettant en avant Fouad Zaban, insiste sur «la ressemblance de l'islam et sa compatibilité avec une culture américaine dominante et conservatrice ${ }^{29}$. » Fouad est un homme qui ne fait de concession ni sur sa foi ni sur son patriotisme. Il donne une vision de l'islam rassurante pour les spectateurs. Ce n'est pas un islam terroriste, mais un islam qui construit, dans le respect de valeurs sociales traditionnalistes.

\section{Conclusion}

Sélection des participants, mise-en-scène des lignes narratives, sélection des images et cadrages sont autant de choix opérés par les concepteurs de All-American Muslim qui nous mènent à faire un parallèle entre ce programme de la chaîne commerciale TLC de la campagne de relation publique lancée par CAIR en $2003^{30}$. S'opposant aux images négatives et stéréotypées diffusées par les médias dans un contexte d'ostracisme des communautés arabes et musulmanes, elles semblent bien poursuivre le même but : « inclure les musulmans dans l'imaginaire de l'Amérique ${ }^{31} »$. Si le parti pris didactique de la campagne de CAIR est conventionnel, il est plus inattendu dans le cadre d'une série de télé-réalité sur une chaîne qui n'est pas (ou plus) à but éducatif. Alsultany insiste sur le peu de succès rencontré par la campagne de CAIR du fait de sa diffusion extrêmement limitée. Le spot de CAIR n'est apparu qu'une fois à la télévision, ses six affiches ne sont parues qu'une seule fois dans le New York Times ${ }^{32}$. De la même façon, l'impact de All-American Muslim sur le public américain n'est pas à surestimer. Le public de la chaîne, celui des femmes de 18 à 49 ans, n'est pas le public jeune et masculin qui consomme les films hollywoodiens grand public qui véhiculent les clichés que la série tente de contrer. De plus, la série n'a pas fait l'audience escomptée et n'a pas

${ }^{28}$ Ibid., p. 145 .

${ }^{29}$ Ibid., p. 148 .

$3^{\circ}$ CAIR a, depuis, lancé d'autres campagnes. En 2009, les spots télévisés «I am Muslim, I am American » mettent en scène des Musulmans de facon individuelle (un policier, une militante des droits de l'homme voilée, un jeune sportif), mais insistent sur des idées d'intégration et de patriotisme par leur slogan « Building Community, Serving Country ». Les images de la campagne «I am Muslim, I am American » peuvent être vues sur ce site à controverse : http://islamizationwatch.blogspot.fr/2009/12/cair-tries-hard-to-promotegood-muslim.html, consulté le 12 février 2014.

${ }^{31}$ Alsultany, p.141

$3^{2}$ En 2012, CAIR choisit de diffuser sa campagne « My Jihad » par des moyens touchant un plus large public : affichage sur des bus et des trains, diffusion sur Facebook, YouTube et Twitter. Ces affiches sont disponibles sur le site officiel Myjihad.org. 
été reconduite à la fin de la première saison. Si la télé-réalité a l'avantage d'offrir une vision de l'intérieur, sans doute le côté didactique du programme a-t-il rebuté le public. Il apparaît cependant que plusieurs séries télévisées de fictions, qui misaient sur l'humour pour lutter contre les stéréotypes, n'aient pas connu plus de succès. Les sitcoms Whoopi! (NBC, 2003-4) et Aliens in America (CBS, 2007-8) n'ont duré qu'une saison. La sitcom canadienne La Petite mosquée dans la prairie (CBC, 2007-2012), si elle a eu du succès dans son pays d'origine, n'a pas rencontré d'écho aux États-Unis. Seul Community (NBC, 2009-) semble tenir la route, mais elle n'inclut qu'un personnage musulman.

Deuxième limite, celle de la représentativité. Pour Alsultany, en insistant sur l'image idéalisée du « bon musulman », la campagne de CAIR exclut de facto de nombreux musulmans, ceux qui ne travaillent pas pour l'Etat, ne sont pas hétérosexuels, ne pratiquent pas leur religion ${ }^{33}$. Ce manque de représentativité est de même l'un des grands reproches fait à All-American Muslim, non seulement par la Florida Association, mais aussi par les musulmans américains eux-mêmes. Ces derniers reprochent au programme le fait qu'il «se concentre uniquement sur une communauté ethnique et religieuse de ciblée (les Libanais Shiites de Dearborn, Michigan) et laisse de côté la majorité des communautés américano-musulmanes, tels les Noirs Américains, les Asiatiques du Sud, les Sunnis et ceux qui appartiennent aux tranches inférieures de la classe moyenne 34 ». Par ce choix très spécifique, la série réduit donc l'image de ce qu'est aujourd'hui un musulman américain. Par ailleurs, dans un effort de contrebalancer des décennies de dénigrement, la série semble parfois verser dans l'extrême inverse. Être patriote ne suffit pas, il faudrait être superpatriote pour être reconnu comme citoyen américain.

Malgré ses paradoxes et ses limites, All-American Muslim apparaît comme une expérience intéressante : s'emparer d'un format de programme à succès, la télé-réalité, afin de réhabiliter une communauté religieuse mal connue et parfois dénigrée. En 2012, TLC récidive avec Breaking Amish, une série de télé-réalité qui se penche sur la communauté Amish. Mais le changement de formule est radical : cette fois, ce sont des membres de la communauté Amish qui quittent leur foyer pour aller vivre à New York, sous le regard de la caméra. Satisfaite du succès de la première saison, TLC enchaîne en 2013 avec une deuxième saison, Breaking Amish: LA, ainsi qu'un spin-off Breaking Amish: Brave New World avec les participants de la première saison. S'il s'agit donc à nouveau d'exploiter le filon religieux,

33 Ibid., p. 148.

34 Wajahat, Ali, "The Reality of the 'All-American Muslim' reality TV show ", Guardian.co.uk, 17 novembre 2011.

http://www.theguardian.com/commentisfree/cifamerica/2011/nov/17/reality-all-

american-muslim-reality-tv, consulté le 30 septembre 2013. 
les a-priori du spectateur ne sont en rien remis en question par les aventures new-yorkaises de ces chrétiens d'un autre temps. Par comparaison, All-American Muslim apparaît donc unique en son genre, polémique par son sujet, mais également expérimental par son dispositif.

\section{L'auteure}

Nolwenn MINGANT est Maître de conférences en civilisation américaine à l'Université Sorbonne nouvelle-Paris 3. Elle a publié en 2010 Hollywood à la conquête du monde : Marchés, stratégies, influences (CNRS Editions). Elle est membre fondateur de l'association CinEcoSa (Cinéma, Economie et Sociétés anglophones) qui œuvre au développement de l'étude de l'économie du cinéma d'un point de vue civilisationniste. Elle est co-fondatrice et secrétaire de rédaction de la revue électronique InMedia, The French Journal of Media and Media Representations in the English-Speaking World (http://inmedia.revues.org/). Elle est porteuse du projet de recherche Cinéma MENA sur les industries cinématographiques du Moyen-Orient et d'Afrique du Nord (http://cinemamena.hypotheses.org/). Ses recherches actuelles portent sur les relations entre Hollywood et la région Afrique du Nord/Moyen-Orient. 